\title{
ESPECIES ÚTILES DEL GÉNERO MIMOSA (Fabaceae-Mimosoideae) en MÉXICO
}

\author{
Sara lucía Camargo-Ricalde, Rosaura Grether, Angélica Martínez-Bernal, \\ Verónica García-García y Saraí Barrios-del-Rosal
}

\author{
Depto. Biología, Div. Ciencias Biológicas y de la Salud, Universidad Autónoma Metropolitana-Iztapalapa \\ Apdo. Postal 55-535; 09340 México, D. F. Tel. 58-04-47-00, fax 58-04-46-88, \\ correo electrónicoslcr@xanum.uam.mx,rogg@xanum.uam.mx,mba@xanum.uam.mx
}

\begin{abstract}
Resumen. Por el número de especies, Mimosa es el género más importante de las Mimosoideae en México; sin embargo, por la ausencia o la dispersión de la información etnobotánica, las especies de este género no han sido consideradas hasta ahora como un recurso natural importante. Con base en la revisión de ejemplares de herbario (CODAGEM, ENCB, IEB, IMSSM, MEXU y UAMIZ), del Banco de Información Etnobotánica de Plantas Mexicanas (BADEPLAM) de la UNAM y de literatura especializada, así como la incorporación de información recabada en el campo, se llevó a cabo una recopilación de los usos de las especies de Mimosa en México y se elaboró una lista con 32 especies que son utilizadas actualmente a nivel local y regional. De éstas, 16 se usan como forraje, 15 tienen uso medicinal, 14 como combustible y 11 son utilizadas para cercas vivas; otros usos menos frecuentes incluyen material para construcción ( 6 especies), melíferas (5), peletería (4), ornamentales (3), comestibles (2) y como implemento agrícola (1).
\end{abstract}

Palabras clave: Fabaceae, México, Mimosa, Mimosoideae, usos.

Abstract. By the number of species, Mimosa is the most important genus of Mimosoideae in Mexico; however, species of this genus have not been considered as important natural resources, due to the lack of ethnobotanic information. Based on the revision of herbarium collections (CODAGEM, ENCB, IEB, IMSSM, MEXU and UAMIZ), of data base of useful plants in Mexico (Banco de Información Etnobotánica de Plantas Mexicanas, BADEPLAM, UNAM), specialized literature, as well as information obtained in the field, a compilation about uses of Mimosa species in Mexico has been done. A list of 32 species with local or regional uses is presented. Of these, 16 are used as fodder, 15 have medicinal use, 14 as fuel, 11 as living fences; other less frequent uses include construction material (6 species), meliferous (5), furriery (4), ornamental (3), edible (2), and agricultural tool (1).

Key words: Fabaceae, Mexico, Mimosa, Mimosoideae, uses.

$\mathbf{E}^{1}$ género Mimosa L. pertenece a la familia Fabaceae, subfamilia Mimosoideae, y se ubica en la tribu Mimoseae. Las leguminosas son una de las seis familias de angiospermas más diversas a nivel mundial y mejor representadas en México (Rzedowski, 1993; Sosa y Dávila, 1994); comprende 650 géneros y alrededor de 18000 especies (Polhill et al., 1981). Los géneros de Mimosoideae con mayor número de especies son: Acacia con 1200-1250, Mimosa con 480-500 e Inga con 300-400 (Elias, 1974, 1981; Sousa y Delgado, 1993).

Mimosa es un género principalmente americano, el $90 \%$ de sus especies se distribuyen del sur de Estados Unidos a la Argentina y el resto se encuentra en África, Asia y Australia (Burkart, 1948; Elias, 1974; Grether, 1978; Lewis y Elias, 1981; Barneby, 1991). En
México, es el género de Mimosoideae mejor representado (104-110 especies), seguido de Acacia con 85 especies. Se considera que nuestro país es el segundo centro de distribución del género después de Brasil (Grether, 1978) con ca. 22\% de las especies, 62 de ellas $(60 \%)$ endémicas (Grether et al., 1996).

La gran diversidad biológica de la familia Fabaceae se refleja en la diversidad de usos de numerosas especies por diferentes culturas a nivel mundial (Isely, 1982; Arellano, 1986). Con base en lo anterior y considerando que Mimosa es el género de Mimosoideae con mayor número de especies en México, el objetivo de este trabajo es registrar y analizar los diversos usos de las especies de este género en el país, con el fin de valorar su importancia como recurso natural. 


\section{Metodología}

Se llevó a cabo la revisión de las colecciones de $\mathrm{Mi}$ mosa de los herbarios CODAGEM, ENCB, IEB, IMSSM, MEXL y LAMIZ. Dado que el registro de los usos de diversas especies de Mimosa se encuentra disperso en la literatura, se hizo una revisión amplia de obras históricas y trabajos etnobotánicos, florísticos y ecológicos y se consultó el Banco de Información Etnobotánica de Plantas Mexicanas (BADEPLAM) del Jardín Botánico, Instituto de Biología, Universidad Nacional Autónoma de México. Asimismo, se incorporó la información sobre usos de Mimosa spp. recabada durante ca. 10 años, en los que se ha realizado trabajo de campo principalmente de las regiones centro, sur y sureste de México, incluyendo los estados de Guanajuato, Querétaro, Michoacán, Tlaxcala, Puebla, Oaxaca, Chiapas, Tabasco, Campeche, Yucatán y Quintana Roo; el material colectado se encuentra depositado en los herbarios IEB, MEXU y UAMIZ.

\section{Resultados y discusión}

Rerisión de obras y trabajos (siglos XVI a XX). Los primeros registros de la utilidad del género Mimosa y en general de la subfamilia Mimosoideae se refieren al uso medicinal. Hasta la fecha esta información es la que se publica con mayor frecuencia.

El registro más antiguo que presenta información sobre el uso medicinal de algunas Mimosoideae, es la obra de De la Cruz (1552) en la que se menciona la planta herbácea llamada "huihuitzyocochizxihuitl", identificada como Mimosa sp. (cuyas hojas eran usadas para aliviar la pérdida o interrupción del sueño); el "tlacoxiloxochitl", que corresponde a Calliandra anomala (Kunth) Macbr; el "mizquitl" o "mezquite", que es Prosopis juliflora (Swartz.) DC. y el "hueynacaztli" o "guanacastle", identificado como Enterolobium cyclocarpum (Jacq.) Griseb., entre otras leguminosas.

Francisco Hernández (1570-1576), en su "Historia de las Plantas de la Nueva España", incluye también algunas Mimosoideae, al igual que Fray Bernardino de Sahagún (1548), en su "Códice Florentino" y Clavijero (1787) en su "Historia Antigüa de Méjico"; sin embargo, en estas obras no se hace mención de ninguna planta que pudiera corresponder al género Mimosa.

Posteriormente, ya a fines del siglo XIX y principios del XX (1870-1910), la Sociedad Mexicana de Historia Natural publicó en su revista La Naturaleza, los usos medicinales de diversas plantas mexicanas, entre ellas seis especies de Mimosoideae. En ese mismo período, en los Anales del Instituto Médico Nacional (1894-1912) se registran solamente dos Mimosoideae y la Nueva Farmacopea Mexicana, editada por la Socie- dad Farmacéutica Mexicana (1904-1952) incluye también seis especies de Mimosoideae. Entre los géneros registrados en estas obras están Acacia, Calliandra, Inga, Leucaena, Lysiloma, Phitecellobium y Prosopis.

Martínez (1969) señala los usos medicinales y algunos datos químicos de 13 especies de Mimosoideae, entre ellas Mimosa purpurascens (= M. distachya var. distachya, según Barneby, 1991). Altschul (1975), a partir de una revisión exhaustiva de las colecciones cle los herbarios de la Universidad de Harvard (A, GH) para sistematizar la información sobre usos de plantas alimenticias y medicinales, registra para México sólo dos especies de Mimosoideae, una de ellas es $M$. palmeri.

En la colección del Herbario Medicinal del Instituto Mexicano del Seguro Social (IMSSM), se encuentran 30 especies de Mimosoideae, entre ellas, cinco especies de Mimosa: M. albida, M. chatocarpa (= M. tricephala), $M$. malacophylla, $M$. pudica, y $M$. recordii (= M. zuatsonii) (Aguilar-Contreras et al., 1994a, 1998).

Por otra parte, los usos populares de algunas especies de Mimosa, por grupos étnicos o por regiones geográficas del país, se encuentran en trabajos etnobotánicos y otros de diversa índole.

Para la región del Bajío y zonas adyacentes, hemos encontrado cuatro especies con usos diversos: $M$. depauperata, $M$. lacerata, $M$. similis y $M$. texana var. texana. Grether (1982) en un estudio ecológico de $M$. biuncifera y $M$. monancistra, en el noroeste del estado de Guanajuato indica sus usos locales.

Camacho-Morfín et al. (1998) proporcionan el primer registro del uso de las ramas de $M$. biuncifera como herramienta para cubrir con tierra las semillas sembradas en los surcos de terrenos de cultivo (rastra) en la región del Valle del Mezquital, Hidalgo.

En el estado de Morelos se han registrado las plantas útiles del Municipio de Tepoztlán, incluyendo a $\mathrm{Mi}$ mosa albida, M. benthamii y M. pudica (Gómez-Salazar y Chong de la Cruz 1985; Cedillo-Portugal 1990).

En el caso de Puebla, Martínez-Bernal y Grether (en prensa) señalan cuatro especies útiles en el Valle de Tehuacán-Cuicatlán: Mimosa albida, M. calcicola, M. luisana y M. polyantha, mientras que Casas et al. (en prensa) registran 104 leguminosas útiles, para el mismo Valle, entre ellas $M$. albida, M. luisana y cuatro ejemplares identificados a nivel genérico y MartínezAlfaro et al. (1995) mencionan también a $M$. albida entre las plantas útiles de la Sierra Norte del estado.

En 1995, Soto y Sousa registraron a $M$. affinis entre las plantas medicinales de la Cuenca del Río Balsas, incluyendo el estado de Michoacán y la zona adyacente de Guerrero.

Browner (1985) en su estudio de las plantas usadas para enfermedades de la reproducción por los chi- 
Cuadro 1. Lista de especies útiles del género Mimosa en México.

Especie

M. acantholoba (Humb. \& Bonpl. ex Willd.) Poir.

${ }^{*}$ M. aculeaticarpa Ortega

are, $\mathrm{a} / \mathrm{cga} / \mathrm{ve}$

M. affinis B. L. Rob.

h,sf/cga/l

M. albida Humb. \& Bonpl. ex Willd. are,art,ard/cga,cgr,cgp,csa,csr,csp/l

M. arenosa Poir.

a, are/ea, er/l

M. bahamensis Benth.

are, a/cga,cgp/l

${ }^{*}$ M. benthamii J. F. Macbr.

are, a/er/l

M. biuncifera Benth.

are/cga/ve

*M. calcicola B. L. Rob.

are/cga/l

${ }^{*} M$. depauperata Benth.

are/cga/l

M. diplotricha C. Wright ex Sauvalle h,sf/cga,cgr/l

M. distachya Cav.

are/ea/l

${ }^{*}$ M. egregia Sandwith

are/ea,er/l

*M. galeottii Benth.

a, are/cga/ve

*M. guatemalensis (Hook. \& Arn.) are,a/ea,er/l Benth.

${ }^{*}$ M. lacerata Rose

${ }^{*} M$. leucaenoides Benth.

*M. Luisana Brandegee

M. malacophylla A. Gray

*M. mollis Benth.

are, $\mathrm{a} / \mathrm{cga} / \mathrm{ve}$
Distribución en México

Sin., Nay., Jal., Col., Mich., Gro., Oax.

N.L., Dgo., Zac., Ags., S.L.P., Gto., Qro., Hgo., Jal., Mich., Edo. Méx., Tlax., Pue., Ver., Gro., Oax., Chis.

Sin., Nay., Jal., Mich., Edo. Méx., Mor., Gro., Oax., Camp., Yuc., Q. Roo.

Sin., Dgo., Tamps., Zac., S.L.P., Gto., Qro., Hgo., Nay., Jal., Col., Mich., Edo. Méx., D.F., Mor., Pue., Ver., Gro., Oax., Tab., Chis., Camp., Yuc., Q. Roo.

Jal., Col., Mich., Oax.

Tab., Camp., Yuc., Q. Roo.

Zac., Ags., Gto., Nay., Jal., Mich., Edo. Méx., Mor., Pue., Gro., Oax.

Son., Chih., Coah., N.L., Tamps., Sin., Dgo., Zac., S.L.P., Gto., Qro., Hgo., Jal., Edo. Méx., D.F., Mor., Tlax., Pue., Ver.

Pue., Ver.

Qro., Hgo.

Nay., Edo. Méx., Pue., Ver., Gro., Oax., Tab., Chis.

B.C.S., Son., Sin., Nay., Jal., Col., Mich., Pue., Yuc.

Mich., Edo. Méx., Gro.

Gto., Nay., Jal., Col., Mich., Edo. Méx., Mor., Pue., Gro., Oax.

Sin., Nay., Jal., Col., Mich., Edo. Méx., Gro.

Qro., Hgo., Jal., Mich., Edo. Méx., Mor., Tlax., Pue., Gro., Oax.

Tamps., S.L.P., Gto., Qro., Hgo.

Pue., Oax.

Coah., N.L., Tamps.

Pue., Mor., Gro., Oax. 
Cuadro 1. Continúa

\begin{tabular}{|c|c|c|}
\hline Especie & $\begin{array}{l}\text { Forma biológica/ } \\
\text { Inflorescencia/fruto }\end{array}$ & Distribución en México \\
\hline M. monancistra Benth. & are/csa/l & $\begin{array}{l}\text { Chih., Coah., N.L., Tamps., Dgo., Zac., } \\
\text { Ags., S.L.P., Gto., Qro., Hgo., Jal., Mich. }\end{array}$ \\
\hline${ }^{*}$ M. palmeri Rose & are, a/ea,er/l & Son., Sin., Nay., Jal., Mich., Gro. \\
\hline M. pigra L. & are/cga,cgr,csa,csr/l & $\begin{array}{l}\text { Sin., Tamps., S.L.P., Nay., Jal., Col., Mich., } \\
\text { Edo. Méx., Mor., Ver., Gro., Oax., Tab., } \\
\text { Chis., Camp., Yuc., Q. Roo. }\end{array}$ \\
\hline M. platycarpa Benth. & are, a/ea/ve & Oax., Chis. \\
\hline${ }^{*} M$. polyantha Benth. & are/ea/l & $\begin{array}{l}\text { Son., Sin., Dgo., Nay., Jal., Mich., Edo. } \\
\text { Méx., Mor., Pue., Ver., Gro., Oax., Chis. }\end{array}$ \\
\hline M. pudica L. & h,sf/cga,cgr,csa,csr/l & $\begin{array}{l}\text { Jal., Col., Mich., Mor., Pue., Ver., Gro., } \\
\text { Oax., Tab., Chis., Camp. }\end{array}$ \\
\hline${ }^{*} M$. similis Britton \& Rose & are/cga,cgr/l & S.L.P., Gto., Qro. \\
\hline${ }^{*}$ M. spirocarpa Rose & are,a/ea/l & Chih., Sin., Col., Mich., Gro. \\
\hline M. tenuiflora (Willd.) Poir. & are,a/ea/l & Oax., Chis. \\
\hline M. texana (A. Gray) Small & are, a/cga/ve & $\begin{array}{l}\text { Son., Coah., N.L., Tamps., Dgo., S.L.P., } \\
\text { Gto., Qro., Hgo., Mich., Pue. }\end{array}$ \\
\hline M. tricephala Cham. \& Schltdl. & are,a/cga,cgr,csa,csr/l & $\begin{array}{l}\text { B.C.S., Mich., Mor., Pue., Ver., Gro., Oax., } \\
\text { Chis. }\end{array}$ \\
\hline $\begin{array}{l}\text { M. watsonii B. L. Rob. } \\
\text { * Endémicas de México }\end{array}$ & b/cgp/l & Ver., Gro., Oax., Tab., Chis. \\
\hline $\begin{array}{l}\text { Simbología: } \\
\text { Forma biológica: } \\
\qquad \begin{aligned} \mathrm{h} & =\text { hierbas } \\
\mathrm{sf} & =\text { sufruticosas } \\
\mathrm{a} & =\text { árboles } \\
\text { are } & =\text { arbustos erectos } \\
\text { ard } & =\text { arbustos decumbentes } \\
\text { art } & =\text { arbustos trepadores } \\
\mathrm{b} & =\text { bejucos }\end{aligned}\end{array}$ & $\begin{aligned} & \text { Tipo de inflorescencias: } \\
& \text { ea }=\text { espigas axilares } \\
& \text { er }=\text { espigas en racimos } \\
& \text { csa }=\text { capítulos subglobosos axilares } \\
& \mathrm{csr}=\text { capítulos subglobosos en racimos } \\
& \mathrm{csp}=\text { capítulos subglobosos en panículas } \\
& \mathrm{cga}=\text { capítulos globosos axilares } \\
& \mathrm{cgr}=\text { capítulos globosos en racimos } \\
& \mathrm{cgp}=\text { capítulos globosos en panículas }\end{aligned}$ & $\begin{array}{l}\text { Tipo de fruto: } \\
I=\text { lomento } \\
\text { ve }=\text { valvas enteras }\end{array}$ \\
\hline
\end{tabular}

nantecos de las tierras altas del Distrito de Ixtlán, Oaxaca, reporta también a $M$. albida.

Camargo-Ricalde et al. (1994) y Camargo-Ricalde (en prensa), a partir del estudio de la biología de $M$. tenuiflora, "tepescohuite", mencionan los diversos usos locales y regionales de esta especie en los estados de Oaxaca y Chiapas, así como el proceso histórico de su comercialización a nivel nacional.

Del Amo (1979) incluye dos especies del género,
M. pudica y $M$. pigra con uso medicinal en el estado de Veracruz.

Para Yucatán, Mendieta y del Amo (1981) citan a M. bahamensis, $M$. pudica y $M$. pigra entre las plantas medicinales del estado. Grether y Camargo-Ricalde (1993) señalan los usos de M. bahamensis en la Península de Yucatán y Camargo-Ricalde y Barrios del Rosal (1997) reportan los usos medicinales de $M$. albida en México. 


\section{Usos}

Las obras históricas señaladas y la revisión sistemática de las colecciones de algunos herbarios (Altschul, 1975; Siri von Reis y Lipp, 1982; Aguilar-Contreras et al., 1994a, 1994b, 1998) registran sólo unas cuantas especies útiles de Mimosa.

En este trabajo se presentan los usos de 32 especies de Mimosa en México ( $31 \%$ de las que se distribuyen en el país), de las cuales 15 (47\%) son endémicas. La mayoría de las especies utilizadas, tienen una amplia distribución en el territorio nacional (cuadro 1).

Las especies del género que se encuentran en México presentan diversas formas biológicas; predominan los arbustos erectos y árboles (69), mientras que 21 especies son herbáceas o sufruticosas, 8 son bejucos y 6 son arbustos decumbentes o trepadores (figura 1a). De ahí que la mayor parte de las especies útiles (figura $1 \mathrm{~b}$ ) sean arbustivas o arbóreas (27) y sólo unas cuantas herbáceas o sufruticosas (3) y bejucos (2).

Las especies de Mimosa se utilizan en diferentes formas: como cerca viva, combustible (leña y/o carbón), comestible, forraje para ganado caprino y ovino principalmente, material para construcción, medicinal, melífera, ornamental, peletería por el alto contenido de taninos principalmente en la corteza y como implemento agrícola. Además, $M$. pudica se usa como planta ceremonial en Tabasco (Romero, 1981).

La mayor parte de las especies son aprovechadas en más de un uso, siendo el forrajero y el medicinal los más frecuentes (cuadros 3 y 4; figura 2); sin embargo, otras especies como $M$. affinis, $M$. diplotricha, $M$. palmeri y $M$. tricephala sólo tienen uso medicinal, mientras que $M$. calcicola, $M$. leucaenoides y $M$. similis sólo son forrajeras, $M$. spirocarpa sólo melífera, $M$. egregia como combustible y $M$. guatemalensis es utilizada para cercas vivas. Con base en la distribución por aparatos y sistemas del cuerpo humano propuesta por Aguilar-Contreras et al. (1994b), de las 15 especies del género con uso medicinal, siete son utilizadas contra enfermedades del aparato digestivo, cinco contra las del aparato urinario, cuatro contra enfermedades y traumatismos de la piel, otras cuatro relacionadas con el sistema nervioso, tres con el aparato reproductor, dos con el sistema óseo y otras dos relacionadas con nosologías tradicionales. Para combatir enfermedades del aparato respiratorio, enfermedades de los ojos y tumores, se registró solamente una especie en cada caso (figura 3).

Destaca la utilización de las hojas y ramas jóvenes como forraje para el ganado caprino y ovino principalmente (16 especies), de leña y carbón como combustible (14 especies), y la formación de cercas vivas alrededor de terrenos de cultivo y potreros (11 especies) (cuadro 3; figura 2). Asimismo, es importante señalar que sólo dos especies son consideradas comestibles: las hojas y los frutos tiernos de $M$. watsonii se consumen en Oaxaca (Arellano, 1986), así como $M$. texana en Guanajuato (sin especificar la parte de la planta utilizada) (figura 2); lo anterior es interesante, ya que muchas de estas plantas tienen un contenido alto de taninos, sugiriendo que no son adecuadas para el consumo humano.

Con base en el trabajo de campo realizado, se puede afirmar que no existe interés por la domesticación

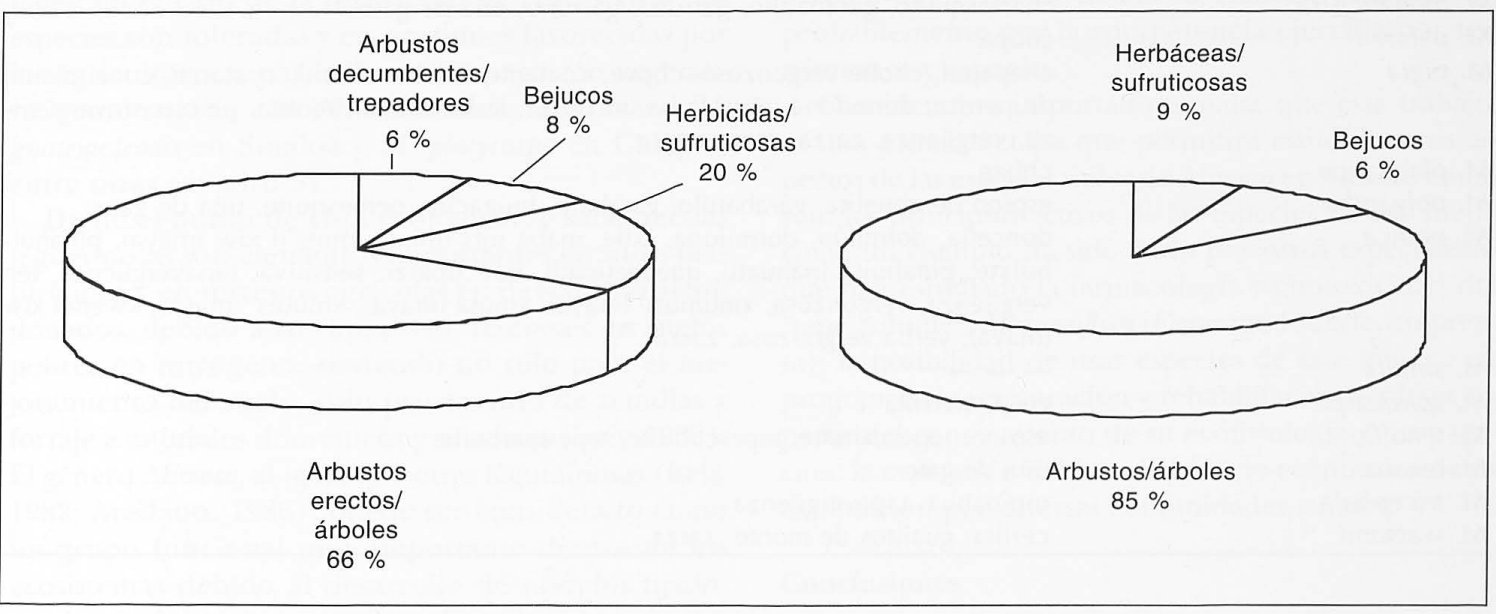

Figura 1. (a) Forma biológica de las especies de Mimosa existentes en México y (b) Forma biológica de las especies útiles de Mimosa en México 
Cuadro 2. Nombres comunes de las especies útiles del género Mimosa en México.

\section{Especie}

M. acantholoba

M. aculeaticarpa

M. affinis
M. albida

M. arenosa

M. bahamensis

M. benthamii

M. biuncifera

M. calcicola

M. depauperata

M. diplotricha

M. distachya

M. egregia

M. galeottii

M. guatemalensis

M. lacerata

M. leucaenoides

M. Luisana

M. malacophylla

M. mollis

M. monancistra

M. palmeri

M. pigra

M. platycarpa

M. polyantha

M. pudica
M. similis
M. spirocarpa
M. tenuiflora
M. texana
M. tricephala
M. watsonii

Nombres comunes

sierrilla, uña de gato, zarza

casirpi, espino, garabatillo, garruño, gatillo, gatuño, quebrachi, quebracho, sashe varudo, sashne varudo, uña de gato

dormilona, sierrilla, vergüenza

ahabiinl (otomí), aha uini, aja muni (otomí), beech (maya), chik chish (tzeltal), chikch'ish (tzeltal), chik ch'ix (tzeltal), ch'ix wamal (tzeltal), cochohuiste, cochohuixtle, cuatantillo, diente de víbora, dormilona, dormilona grande, espina, espinita dormilona, espinosa, iñun dú-a (mixteco), jeech (maya), kuan chev, lojon chijo bomol (tzotzil), lotom chij bomol (tzotzil), lot'om ch'ix jomol (tzeltal), lotz' om chij (tzotzil), mala hierba, oo-tú-cu (chinanteco), palo de espino, pinahuiste, pinahuitz (náhuatl), pinahuitztli, rosa concha, sakil t'om ch'ix (tzeltal), sensitiva, sierrilla negra, sinvergüenza, tapavergüenza, tzajal lotz'om (tzotzil), tzajal tuxnuk'te (tzotzil), tz'amte ch'en pom, uña de gato, uña de pájaro, vergonzosa, vergüenza, yech din, yucundita-ha (mixteco), zarcilla, zarza, zarzaparilla

cucharita del cerro, espino, huizache, timbre, uña de gato

boxcatzim, catzim, kaatsim (maya), katsim, kitsim, motita morada, sak-katsim, sak-katzim, saskatzim, zaccatzin, zas-katzin

espina de herrero, espino herrero, espinorrero, garabatillo, garruño, hebrero, herrero, palo herrero, quilahuacate, tecolhuixtle, tecolohuixtle, tehuixtle, uña de gato

casirpi, garabatillo, garruño, gatillo, gatuño, huiscalate, huizache, huizachito, shaminí (otomí), shashne, uña de gato

uña de gato

gatillo, huizache, uña de gato

cuatantillo, rascapetate, sierrilla, sierrilla chica, tz'abab ch'ix (tzotzil), zarza

cuca, cuilón, cuillón, iguano, uña de gato

chacapo, gavilancillo, sacapo, uña de gato

carispe, espino, espinorrero, espinosa, ixextimia, tehuistle, uña de gato

cuilón, espina herrero, juilón

facalhuixtle, garabato, mezquite, tecolhuistle, uña de gato

chaparro prieto, chicharrillo, quiebra fierro, quiebra machete

cumito

charrasquillo, raspahuevos, raspilla

cuajiotal, garabato, pipinahua

charrasquillo, garabatillo, garruña, garruño, uña de gato

chopa

chapapul, chobe vergonzoso, chove, coatante, cochiz-xíhuitl, cuatante, cuca, diente de perrito, dormilona, iñun-duba (mixteco), kuka (maya), motita, palote, sinvergüenza, vergüenza, zarza, zarza negra

chixte

espino de abuelita, garabatillo, garabato, huizache, pemesquite, uña de gato doncella, dormilón, dormilona, ixtle, maba ujts (mixe), muts'il xiw (maya), pinahuihuixte, pinahuitz (náhuatl), quececupatli, quecupatzi, sensitiva, tapavergüenza, ten vergüenza, vergonzosa, xmumuts' (maya), xmutx (maya), xmuuts' (maya), xwenel xiw (maya), yerba vergonzosa, zarza

\section{- - -}

cuca, sierrilla

espino, tepescahuite, tepescohuite, tepesquehuite

uña de gato

pipinahua, tapavergüenza

cerilla, guajitos de monte, zarza 


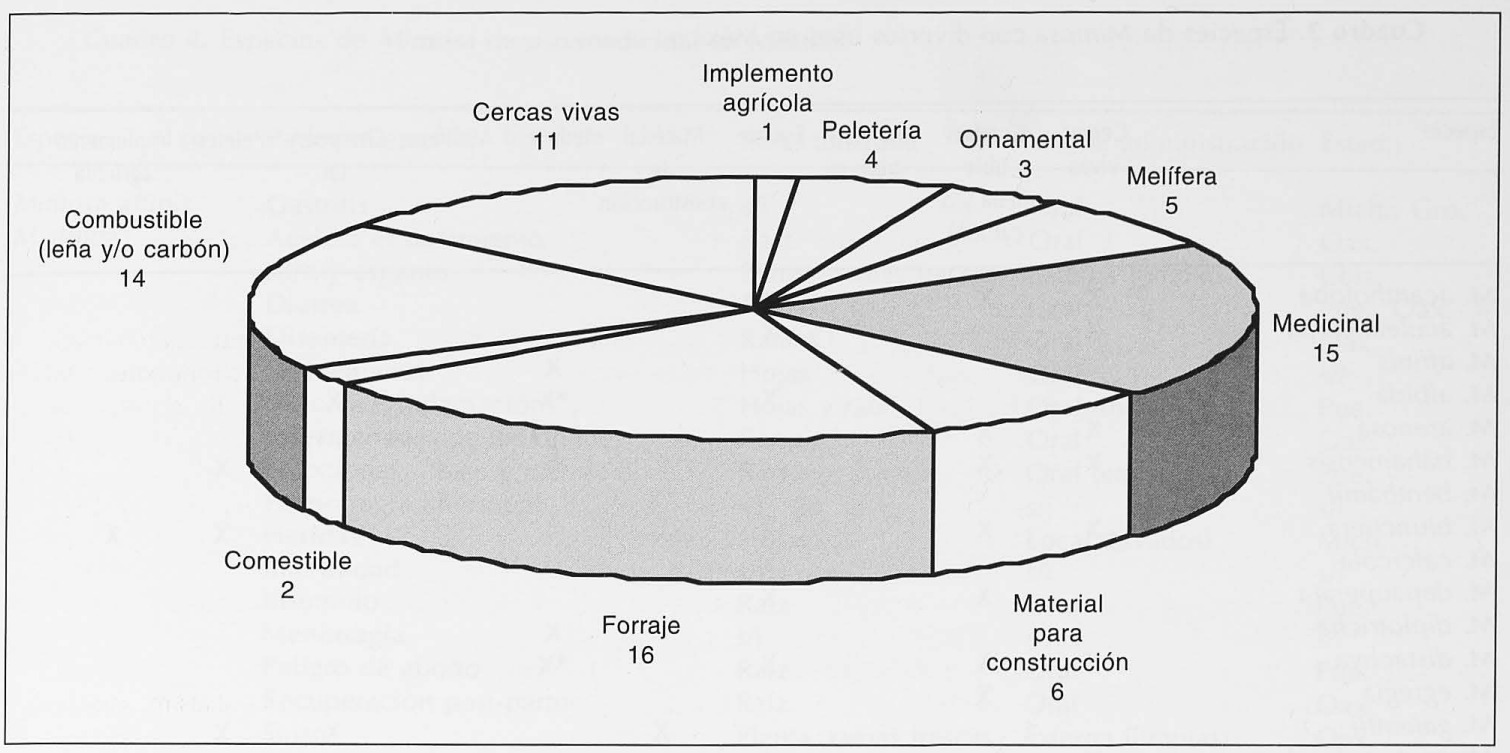

Figura 2. Número de especies de Mimosa con distintos usos en México.

de alguna de las especies de este estudio, con excepción del "tepescohuite", Mimosa tenuiflora, ya que los agricultores toleran su presencia y favorecen su desarrollo en terrenos de cultivo en descanso y abandonados para comercializar su corteza y para la regeneración del suelo (Camargo-Ricalde, en prensa). Algunas especies son utilizadas in situ como ocurre con las forrajeras y otras son recolectadas para ser usadas posteriormente, ya sea frescas, secas o con algún tipo de procesamiento como sucede con las medicinales; en el caso de la formación de cercas vivas, las especies son toleradas y en ocasiones favorecidas por los agricultores a partir de su propagación vegetativa, como sucede con $M$. acantholoba en Oaxaca, $M$. guatemalensis en Sinaloa y $M$. platycarpa en Chiapas, entre otras (cuadro 3).

Desde el punto de vista ecológico, se sabe que las leguminosas son elementos importantes en sitios perturbados y en terrenos agrícolas en descanso o abandonados, debido a su capacidad de crecer en suelos pobres en nitrógeno, sirviendo no sólo para el mejoramiento del suelo, sino proveyendo de semillas y forraje a animales domésticos y silvestres (Isely, 1982). El género Mimosa, al igual que otras leguminosas (Isely, 1982; Arellano, 1986), puede ser considerado como un grupo funcional muy importante dentro de los ecosistemas debido al desarrollo de nódulos fijadores de nitrógeno en sus raíces al asociarse con bacterias del género Rhizobium, lo que les da la capacidad de enriquecer el suelo. Además, se conoce el papel de $M$. luisana como "nodriza" del "tetecho", Neobuxbaumia tetetzo, (Valiente-Banuet et al., 1991) y el caso de varias especies, como Mimosa bahamensis, M. biuncifera, $M$. monancistra y $M$. tenuiflora, que juegan un papel importante como especies oportunistas y típicamente secundarias al colonizar sitios perturbados, resultantes de actividades humanas que crean las condiciones favorables para la invasión y desarrollo de estas especies (Camargo-Ricalde et al., 1995). Sin embargo, Mimosa albida ha sido reportada como especie nociva para los cultivos en el norte de Puebla (Basurto-Peña, 1982), probablemente por la competencia ejercida por nutrimentos y espacio.

Consideramos importante señalar que este trabajo, es una primera etapa que permitirá estudiar otros aspectos de las especies útiles de Mimosa en México como son: los principios actiros de las especies de uso medicinal, un ejemplo ha sido dado por otros especialistas que han estudiado la farmacología y citotoxicidad del "tepescohuite", M. tenuiflora (Camargo-Ricalde, en prensa); la posibilidad de usar especies de este género en programas de restauración y rehabilitación ecológica a partir del conocimiento de su ecofisiología y propagación; la evaluación del beneficio socio-económico de su explotación por diversas comunidades rurales.

\section{Conclusiones}

Por el número de especies conocidas de la subfamilia Mimosoideae en México, el género Mimosa es el 
Cuadro 3. Especies de Mimosa con diversos usos en México.

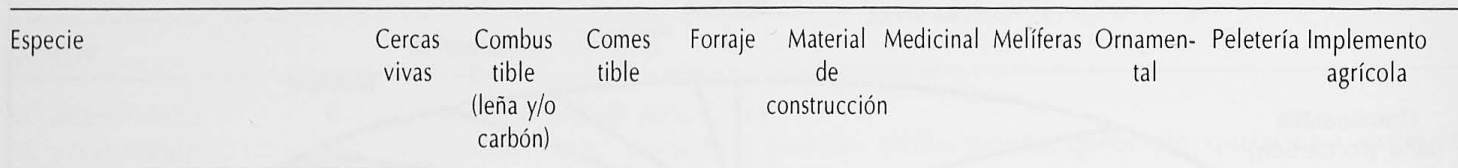

\begin{tabular}{|c|c|c|c|c|c|c|c|c|c|c|}
\hline M. acantholoba & $x$ & $x$ & & $x$ & & & & & & \\
\hline M. aculeaticarpa & $x$ & & & $x$ & & & & & & \\
\hline M. affinis & & & & & & $x$ & & & & \\
\hline M. albida & & & & $x$ & & $* X$ & & $x$ & & \\
\hline M. arenosa & $x$ & $x$ & & & & & & & & \\
\hline M. bahamensis & $x$ & $x$ & & & $x$ & $* X$ & $x$ & & $x$ & \\
\hline M. benthamii & & & & & $* X$ & & $x$ & & & \\
\hline M. biuncifera & $x$ & $x$ & & $x$ & $x$ & & & & $x$ & $x$ \\
\hline M. calcicola & & & & $x$ & & & & & & \\
\hline M. depauperata & & $x$ & & $x$ & & & & & & \\
\hline M. diplotricha & & & & & & $x$ & & & & \\
\hline M. distachya & $x$ & $x$ & & $x$ & & $* x$ & & & & \\
\hline M. egregia & & $x$ & & & & & & & & \\
\hline M. galeottii & & & & & $x$ & & & $x$ & $x$ & \\
\hline M. guatemalensis & $x$ & & & & & & & & & \\
\hline M. lacerata & & $x$ & & $x$ & & $x$ & & & & \\
\hline M. leucaenoides & & & & $x$ & & & & & & \\
\hline M. luisana & $x$ & $x$ & & $x$ & $x$ & & & & & \\
\hline M. malacophylla & & & & $x$ & & $x$ & & & & \\
\hline M. mollis & & & & $x$ & & & & $x$ & & \\
\hline M. monancistra & & $x$ & & $x$ & $x$ & & & & & \\
\hline M. palmeri & & & & & & $x$ & & & & \\
\hline M. pigra & & $x$ & & & & $x$ & $x$ & & & \\
\hline M. platycarpa & $x$ & & & & & $x$ & & & & \\
\hline M. polyantha & $x$ & $x$ & & $x$ & & & & & & \\
\hline M. pudica & & & & & & ${ }^{*} X$ & $* x$ & & & \\
\hline M. similis & & & & $x$ & & & & & & \\
\hline M. spirocarpa & & & & & & & $x$ & & & \\
\hline M. tenuiflora & $x$ & $x$ & & & & $* x$ & & & $x$ & \\
\hline M. texana & & $x$ & $x$ & $x$ & & $x$ & & & & \\
\hline M.tricephala & & & & & & $x$ & & & & \\
\hline M. watsonii & & & $x$ & & & $x$ & & & & \\
\hline * BADEPLAM (2000) & & & & & & & & & & \\
\hline
\end{tabular}

más importante; sin embargo, es un género que ha sido poco estudiado desde el punto de vista biológico, ecológico y etnobotánico en el país.

De las 104-110 especies de Mimosa que crecen en el país, $32(31 \%)$ son utilizadas de diversas formas por comunidades rurales e indígenas, a lo largo del territorio nacional.

Los usos observados con mayor frecuencia son el forrajero (16 especies) y el medicinal (15 especies).

La mayoría de las especies son utilizadas al menos en dos formas distintas, mientras que otras como $M$. affinis, $M$. calcicola, $M$. diplotricha, $M$. egregia, $M$. guatemalensis, $M$. leucaenoides, M. palmeri, $M$. similis, $M$. spirocarpa y $M$. tricephala, sólo tienen un uso específico.
En este trabajo no se ha encontrado una relación evidente entre los nombres comunes y los usos de las especies estudiadas; aparentemente estos nombres se refieren a alguna característica de las plantas.

Se conocen numerosos nombres comunes para las especies de Mimosa con usos populares. Entre ellos destaca el nombre "uña de gato", empleado para 15 de las 32 especies estudiadas, haciendo referencia a sus aguijones recurvados, aunque la mayoría de ellas también reciben otros nombres vulgares.

Como recurso natural el género es importante ya que cubre diversas necesidades de la población rural. Sin embargo estas plantas no se comercializan, sino que se utilizan in situ o son recolectadas para con- 
Cuadro 4. Especies de Mimosa de uso medicinal en México.

\begin{tabular}{|c|c|c|c|c|}
\hline Especie & Padecimiento & Parte utilizada & Vía de administración & Estado \\
\hline Mimosa affinis & Gastritis & $s / i$ & Oral & Mich., Gro. \\
\hline \multirow[t]{17}{*}{ M.albida } & Acelera el nacimiento & Raíz & Oral & Oax. \\
\hline & Aire y espanto & Planta, ramas frescas & Externa (limpias) & Chis. \\
\hline & Diarrea & Ramas & Oral & Pue., Oax. \\
\hline & Disentería & Ramas & Oral & Oax. \\
\hline & Dismenorrea & Hojas & Oral & $s / i$ \\
\hline & Dolores e inflamación & Hojas y raíz & Oral (infusión) & Pue. \\
\hline & Enfermedades de los riñones & Ramas & Oral & Oax. \\
\hline & Erupciones, fuego y mal de boca & Raíz & Oral (enjuagues) & Pue. \\
\hline & Hemorragia menstrual & $s / i$ & $s / i$ & $s / i$ \\
\hline & Heridas & Hojas & Local (lavados) & Mich. \\
\hline & Infertilidad & $s / i$ & $s / i$ & $s / i$ \\
\hline & Insomnio & Raíz & Oral & Pue. \\
\hline & Menorragia & $s / i$ & $s / i$ & $s / i$ \\
\hline & Peligro de aborto & Raíz & Oral & Pue. \\
\hline & Recuperación post-parto & Raíz & Oral & Oax. \\
\hline & Susto & Planta, ramas frescas & Externa (limpias) & Oax. \\
\hline & Vista nublada & Raíz & Local & Pue. \\
\hline \multirow[t]{2}{*}{ M. bahamensis } & Disentería y mal de orín & Raíz & Oral & Yuc. \\
\hline & Dolor de cabeza & Raíz & Oral & Yuc. \\
\hline M. diplotricha & Mal de orín y tumores & $s / i$ & Oral & $s / i$ \\
\hline \multirow[t]{2}{*}{ M. distachya } & Endurecer las encías & Corteza & Masticada & B.C., Son., Sin. \\
\hline & Exceso de grasa y heridas de la piel & Tallo y corteza & Local & $s / i$ \\
\hline M. lacerata & Erupciones en la boca y fuegos & $\mathrm{s} / \mathrm{i}$ & $\mathrm{s} / \mathrm{i}$ & $s / i$ \\
\hline M. malacophylla & Enfermedades de los riñones & Planta completa & Oral (infusión) & N.L. \\
\hline M. palmeri & Debilidad de las encías & Corteza & Masticada & $s / i$ \\
\hline \multirow[t]{6}{*}{ M. pigra } & Debilidades de las encías & Flor & Oral (enjuagues) & Ver., Yuc. \\
\hline & Diarrea, disentería & Flor & Oral & Ver., Yuc. \\
\hline & Enfermedades de la garganta & Flor & Local (enjuagues) & Ver., Yuc. \\
\hline & Enfermedades de los riñones & Raíz & Oral & Ver., Yuc. \\
\hline & Flujos blancos & Flor & Local (baños) & Ver., Yuc. \\
\hline & Irritación de la boca & Flor & Local (enjuagues) & Ver., Yuc. \\
\hline M. platycarpa & Aire & $s / i$ & $s / i$ & $s / i$ \\
\hline \multirow[t]{4}{*}{ M. pudica } & Ansiedad y nerviosismo & Hoja & $\begin{array}{l}\text { Externa (baños) } \\
\text { (sedante, narcótico) }\end{array}$ & $s / i$ \\
\hline & Epilepsia & Hoja & Oral & Ver. \\
\hline & Fatiga & Hoja & Oral (estimulante) & Ver. \\
\hline & Insomnio & Raíz y tallo & Se fuman (soporífero) & $s / i$ \\
\hline \multirow[t]{3}{*}{ M. tenuiflora } & Enfermedades gastrointestinales & Corteza & Oral & Oax., Chis. \\
\hline & $\begin{array}{l}\text { Escoriaciones en la boca, } \\
\text { encías, paladar }\end{array}$ & Corteza & Oral (enjuagues) & Oax., Chis. \\
\hline & Heridas y quemaduras de la piel & Corteza & $\begin{array}{l}\text { Local (compresas y } \\
\text { pomadas) }\end{array}$ & Oax., Chis. \\
\hline M. texana & $\begin{array}{l}\text { Cansancio, dolor de cabeza, } \\
\text { fiebre y reumatismo }\end{array}$ & $s / i$ & $s / i$ & Gto. \\
\hline M. tricephala & Desarrollo & Planta & $\begin{array}{l}\text { Oral (infusión) } \\
\text { Local (cataplasma) }\end{array}$ & Ver. \\
\hline M. watsonii & Gonorrea & Raíz & Oral & Oax. \\
\hline $\mathrm{s} / \mathrm{i}=\sin$ información & & & & \\
\hline
\end{tabular}


Figura 3. Esquema que ilustra las especies de Mimosa que son utilizadas en la medicina tradicional, agrupadas con relación a los aparatos y sistemas del cuerpo humano.

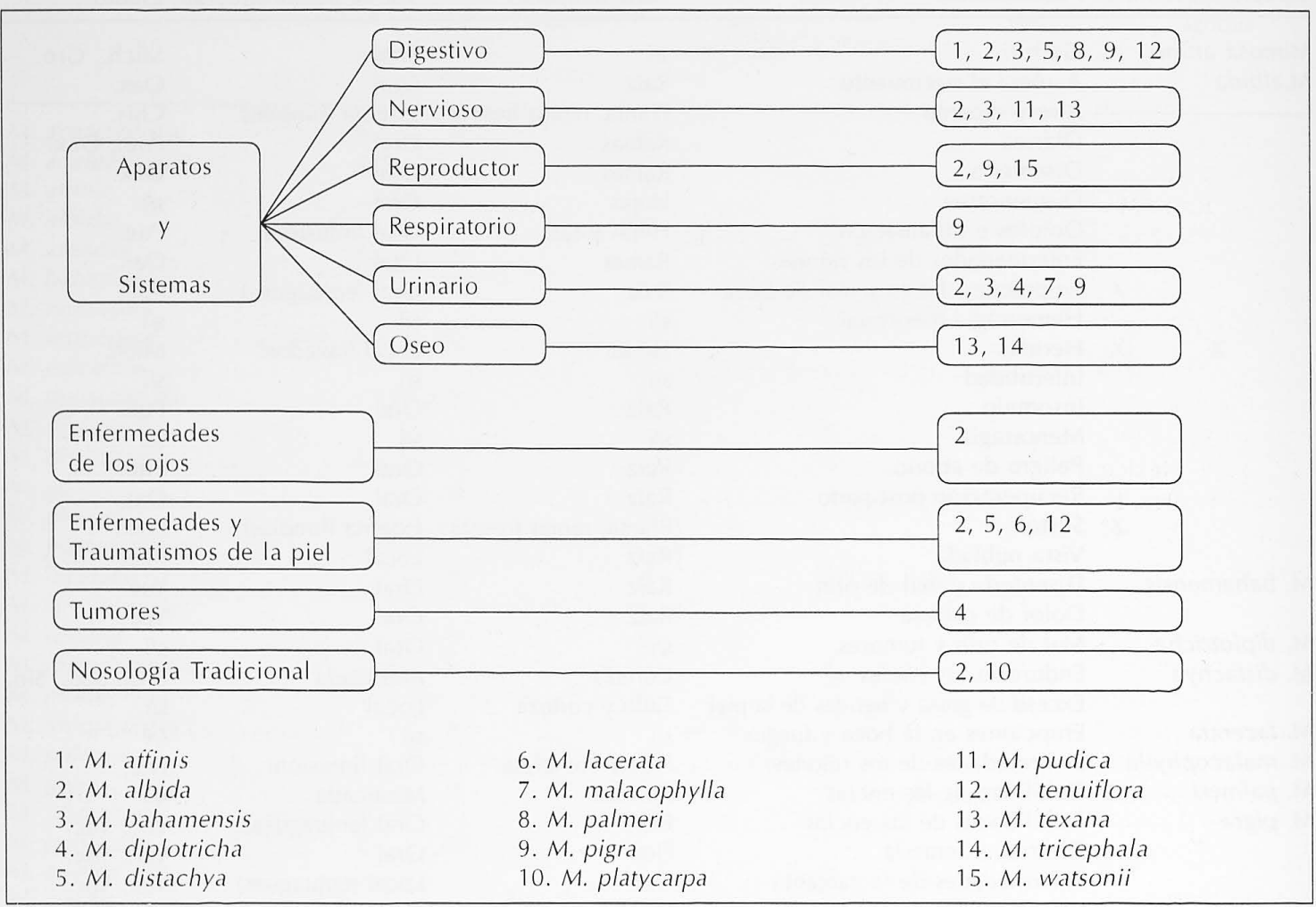

sumo familiar y/o comunitario, por lo que no hay beneficios económicos directos. En este último caso, la única excepción conocida es el "tepescohuite", $M$. tenuiflora, que ha generado una industria cosmética a nivel nacional e internacional y que ya cuenta con el registro de dos patentes relacionadas con la actividad biológica de su corteza (NAPRALERT, 2000).

Consideramos que se requieren estudios bioquímicos, de biología reproductiva, ecológicos, etnobiológicos y genéticos de las especies de este género, ya que en la medida en que se conozcan estos recursos vegetales, será posible incorporarlos en el diseño de estrategias de manejo y de conservación en forma. sustentable.

\section{Agradecimientos}

A la M. en C. Abigaíl Aguilar Contreras, curadora del herbario del Instituto Mexicano del Seguro Social (IMSSM) por la revisión crítica del manuscrito, y al Dr. Javier Caballero y a la Biol. Laura Cortés, del Jar- dín Botánico, Instituto de Biología, LNAM, por permitirnos consultar el BADEPLAM. Al CONACyT 112386/ 121585 y a la Universidad Agrícola de Noruega (NLH).

\section{Literatura citada}

Aguilar-Contreras A., Camacho J.R., Chino S., Jácquez P. y López M.E. 1994a. Herbario Medicinal del Instituto Mexicano del Seguro Social: Información Etnobotánica. Instituto Mexicano del Seguro Social. México. 253 p.

Aguilar-Contreras A., Camacho J.R., Chino S., Jácquez P. y López M.E. 1994b. Plantas Medicinales del Herbario del IMSSS. Cuadros Básicos por Aparatos y Sistemas del Cuerpo Humano. Instituto Mexicano del Seguro Social. México. 218 p.

Aguilar-Contreras A., Camacho-Puliclo J.R., Chino-Vargas S., Jácquez-Ríos P. y López-Villafranco M.E. 1998. Plantas Medicinales del Herbario IMSS: su distribución por enfermedades. Instituto Mexicano del Seguro Social y Grupo Roche Syntex de México, S.A. de C.V. México. 167 p. Altschul A.M. 1975. Drugs and foods from little known plants. 
Notes in Harvard University Herbaria. Harvard University Press, Cambridge, 366 p.

Arellano J. 1986. Etnobotánica de leguminosas: notas sobre algunas de las especies utilizadas en la alimentación en México. Anales del Instituto de Biología 57:123-142.

BADEPLAM (Banco de Información Etnobotánica de Plantas Mexicanas). Caballero J. (resp.) y Cortés L. (admon.). Jardín Botánico, Instituto de Biología, UNAM. Revisión: 2000.09.25.

Barneby R.C. 1991. Sensitivae Censitae. A description of the genus Mimosa L. (Mimosaceae) in the New World. Memoirs of the New York Botanical Garden 65:1-835.

Basurto-Peña F. 1982. Huertos familiares en dos comunidades nahuas de la Sierra Norte de Puebla: Yancuictlalpan y Cuauhtapanaloyan. Tesis de Licenciatura, Facultad de Ciencias, Unam, México, 140 pp.

Browner C.H. 1985. Plants used for reproductive health in Oaxaca, Mexico. Economic Botany 39:482-504.

Burkart A. 1948. Las especies de Mimosa de la Flora Argentina. Darwiniana 8:11-231.

Camacho-Morfín D.C., Sandoval C. y Ayala A. 1998. Identificación de especies leñosas forrajeras en el Valle del Mezquital, Hidalgo, México. Libro de Resúmenes. VII Congreso Latinoamericano y XIV Congreso Mexicano de Botánica. México. 57.

Camargo-Ricalde S.L., Grether R. y Martínez-Bernal A. 1994. Uso medicinal del "tepescohuite", Mimosa tenuiflora (Leguminosae), en México. ContactoS, UAM-Iztapalapa 5:2934.

Camargo-Ricalde S.L., Grether R. y Martínez-Bernal A. 1995. Cuatro especies oportunistas del género Mimosa (Leguminosae) en México. ContactoS, UAM-Iztapalapa 10:5-15.

Camargo-Ricalde S.L. y Barrios-del-Rosal S. 1997. Uso medicinal de Mimosa albida Humb. \& Bonpl. ex Willd. (Leguminosae) en México. ContactoS, UAM-Iztapalapa 23:5-11.

Camargo-Ricalde S.L. 2000 Descripción, distribución, anatomía, composición química y usos de Mimosa tenuiflora (Fabaceae-Mimosoideae) en México. Revista de Biologia Tropical 48 (4):939-954.

Casas A., Valiente-Banuet A., Viveros J.L., Caballero J., Cortés L., Dávila P., Lira R. y Rodríguez I. Plant resources of the Tehuacán-Cuicatlán Valley, Mexico. Economic Botany (en prensa).

Cedillo-Portugal E. 1990. Las plantas útiles del Municipio de Tepoztlán, Morelos. Tesis de Maestría en Ciencias, Facultad de Ciencias, UNAM, México, 425 pp.

Clavijero F.J. 1787. Historia antigua de Méjico. Diez libros sobre la tierra, animales y habitantes de Méjico. (Ed. 1974). Talleres de Litográfica y Tipográfica Yolva, S.A. México. 439 p.

De la Cruz M. 1552. Libellus de Medicinalibus Indorum Herbis. Manuscrito, (Ed. 1964), versión española. Instituto Mexicano del Seguro Social. México. 385 p.
Del Amo S. 1979. Plantas medicinales del estado de Vearacruz. Instituto Nacional de Investigaciones sobre Recursos Bióticos (INIREB). México. 279 p.

Elias T.S. 1974. The genera of Mimosoideae (Leguminosae) in the southeastern United States. Journal of the Arnold Arboretum 55: 83 .

Elias T.S. 1981. Mimosoideae. En: Polhill R.M. y Raven P.H. Eds. Advances in Legume Systematics. Part 1. Royal Botanic Gardens, Kew, England, pp. 143-151.

Gómez-Salazar L. y Chong de la Cruz E. 1985. Cocimiento y usos de la flora de Amatlán, Municipio de Tepoztlán, Morelos. Tesis de Licenciatura, Facultad de Ciencias, UNAM, México, 185 pp.

Grether R. 1978. A general review of the genus Mimosa in México. Bulletin of the International Group for the Study of Mimosoideae 6:45-50.

Grether R. 1982. Aspectos ecológicos de Mimosa biuncifera y Mimosa monancistra en el noroeste del estado de Guanajuato. Boletin de la Sociedad Botánica de México 43:4360.

Grether R. y Camargo-Ricalde S.L. 1993. Mimosa bahamensis (Leguminosae) en la Península de Yucatán. Boletin de la Sociedad Botánica de México 53:55-72.

Grether R., Camargo-Ricalde S.L. y Martínez-Bernal A. 1996. Especies del género Mimosa (Leguminosae) presentes en México. Boletin de la Sociedad Botánica de México 58:149152.

Hernández F. 1570-1576. Historia de las plantas de la Nueva España. (Ed. 1943). Tomo 2: 560-561.

Isely D. 1982. Leguminosae and Homo sapiens. Economic Botany $36: 46-70$.

Lewis G.P. y Elias T.S. 1981. Tribu Mimoseae. En: Polhill R.M. y Raven P.H. Eds. Advances in Legume Systematics. Part 1. Royal Botanic Gardens, Kew, England, pp. 155168.

Martínez-Alfaro M.A., Evangelista V., Mendoza M., Morales G., Toledo G. y Wong A. 1995. Catálogo de plantas útiles de la Sierra Norte de Puebla, México. Instituto de Biología, UNAM. México. 303 p.

Martínez-Bernal A. y Grether R. Mimosa. En: Kelly L. y Medina-Lemos R. Eds. Flora del Valle de Tehuacán-Cuicatlán. Fascículo 33. Instituto de Biología, Universidad Nacional Autónoma de México. México (en prensa).

Martínez M. 1969. Las plantas medicinales de México. Botas. México. 656 p.

Mendieta R.M. y Del Amo S. 1981. Plantas medicinales del estado de Yucatán. Instituto Nacional de Investigaciones sobre Recursos Bióticos (INIREB). C.E.C.S.A. México. 428 p.

NAPRALERT $^{\text {SM }}$ (Natural Products Alert). Departamento de Química Médica y Farmacognosis, Colegio de Farmacia de la Universidad de Illinois en Chicago. NAPRALERT. Visitado 2000.09.26. URL: http://www.ag.uiuc.edu/ ffh/ napra.html. 
Polhill R.M., Raven P.H. y Stirton C.H. 1981. Evolution and Systematics of the Leguminosae. En: Polhill R.M. y Raven P.H. Eds. Advances in Legume Systematics. Part 1. Royal Botanic Gardens, Kew, England, pp. 1-26.

Romero C. 1981. Etnobotánica de los huertos familiares en los Ejidos Habaneros 2a. Sección de H. Cárdenas y Mantilla de Cunduacán, Tabasco. Tesis de Licenciatura, Facultad de Ciencias, UNAM, México, 226 pp.

Rzedowski, J. 1993. Diversity and origins of the phanerogamic flora of Mexico. En: Ramamoorthy T. P., Bye R., Lot A. y Fa J. Eds. Biological Diversity of Mexico: origins and distribution. Oxford University Press, pp. 129-144.

Sahagún F.B. de. 1548. Códice Florentino. (Ed. 1979). Vol. III. Libros Décimo y Undécimo. Fo: 97-114 y 139-178. Reproducción Facsimilar, Secretaría de Gobernación, México.

Siri von R. y Lipp F.J. 1982. New plant sources for drogs and foods from the New York Botanical Garden Herbarium. Harvard University Press, Cambridge, 362 pp.

Sosa V. y Davila P. 1994. Una evaluación del conocimiento florístico de México. Annals of the Missouri Botanical Garden 81: 749-757.

Soto-Núñez J.C. y Sousa M. 1995. Plantas Medicinales de la Cuenca del Río Balsas. Instituto de Biología, UNAM. México. 198 p.

Sousa M. y Delgado A. 1993. Mexican Leguminosae: phytogeography, endemism, and origins. En: Ramamoorthy T.P., Bye R., Lot A. y Fa J. Eds. Biological diversity of Mexico: origins and distribution. Oxford University Press, pp. $459-511$.

Valiente-Banuet A., Vite F. y Zavala-Hurtado A. 1991. Interaction between the cactus Neobuxbaumia tetetzo and the nurse shrub Mimosa luisana. Journal of Vegetation Science 2:11-14. 